OPEN ACCESS

Edited by:

Eric Cascales,

Aix-Marseille Université, France

Reviewed by:

Andreas Diepold,

Max Planck Institute for Terrestrial

Microbiology, Germany

Kelly Hughes,

The University of Utah, United States

${ }^{*}$ Correspondence:

Julien R. C. Bergeron

julien.bergeron@kcl.ac.uk

tPresent address:

Giuseppina Mariano,

Microbes in Health and Disease

Theme, Newcastle University

Biosciences Institute, Newcastle

University, Newcastle upon Tyne,

United Kingdom

Raquel Faba-Rodriguez,

Peak Proteins Ltd., Mereside, United Kingdom

Specialty section:

This article was submitted to Microbial Physiology and Metabolism, a section of the journal

Frontiers in Microbiology

Received: 23 September 2021

Accepted: 08 December 2021

Published: 11 January 2022

Citation:

Mariano G, Faba-Rodriguez R, Bui S,

Zhao W, Ross J, Tzokov SB and

Bergeron JRC (2022) Oligomerization

of the FliF Domains Suggests a

Coordinated Assembly of the Bacterial Flagellum MS Ring.

Front. Microbiol. 12:781960.

doi: 10.3389/fmicb.2021.781960

\section{Oligomerization of the FliF Domains Suggests a Coordinated Assembly of the Bacterial Flagellum MS Ring}

\author{
Giuseppina Mariano ${ }^{1,2 \dagger}$, Raquel Faba-Rodriguez ${ }^{2 \dagger}$, Soi Bui ${ }^{1}$, Weilong Zhao ${ }^{1}$, James Ross ${ }^{3}$, \\ Svetomir B. Tzokov ${ }^{2}$ and Julien R. C. Bergeron ${ }^{1,2 *}$ \\ ${ }^{1}$ Randall Division of Cell and Molecular Biophysics, King's College London, London, United Kingdom, ${ }^{2}$ Department of \\ Molecular Biology and Biotechnology, University of Sheffield, Sheffield, United Kingdom, ${ }^{3}$ Department of Biochemistry, \\ University of British Columbia, Vancouver, BC, Canada
}

The bacterial flagellum is a complex, self-assembling macromolecular machine that powers bacterial motility. It plays diverse roles in bacterial virulence, including aiding in colonization and dissemination during infection. The flagellum consists of a filamentous structure protruding from the cell, and of the basal body, a large assembly that spans the cell envelope. The basal body is comprised of over 20 different proteins forming several concentric ring structures, termed the $\mathrm{M}$ - S- L- P- and C-rings, respectively. In particular, the MS rings are formed by a single protein FliF, which consists of two trans-membrane helices anchoring it to the inner membrane and surrounding a large periplasmic domain. Assembly of the MS ring, through oligomerization of FliF, is one of the first steps of basal body assembly. Previous computational analysis had shown that the periplasmic region of FliF consists of three structurally similar domains, termed Ring-Building Motif (RBM)1, RBM2, and RBM3. The structure of the MS-ring has been reported recently, and unexpectedly shown that these three domains adopt different symmetries, with RBM3 having a 34-mer stoichiometry, while RBM2 adopts two distinct positions in the complex, including a 23-mer ring. This observation raises some important question on the assembly of the MS ring, and the formation of this symmetry mismatch within a single protein. In this study, we analyze the oligomerization of the individual RBM domains in isolation, in the Salmonella enterica serovar Typhimurium FliF ortholog. We demonstrate that the periplasmic domain of FliF assembles into the MS ring, in the absence of the transmembrane helices. We also report that the RBM2 and RBM3 domains oligomerize into ring structures, but not RBM1. Intriguingly, we observe that a construct encompassing RBM1 and RBM2 is monomeric, suggesting that RBM1 interacts with RBM2, and inhibits its oligomerization. However, this inhibition is lifted by the addition of RBM3. Collectively, this data suggest a mechanism for the controlled assembly of the MS ring.

Keywords: bacteria, flagellum, Salmonella, cryo-EM, structure 


\section{INTRODUCTION}

The flagellum is a complex macromolecular motor, whose role is to allow swimming motility, through the rotation of a long filament at the bacterium cell surface. The flagellum is employed by many bacteria to swim in liquid environments (Minamino and Imada, 2015), but it also represents an important virulence factor, playing central roles in cell adhesion and invasion, secretion of other virulence factors, and biofilm formation (Duan et al., 2013). The bacterial flagellum can be divided into four major regions: Embedded in the inner membrane, the rotor and stator complexes are responsible for inducing filament rotation, using the proton motor force or sodium gradient (depending on the bacterial species; Berg, 2003; Li et al., 2011). The basal body is the region that spans the cell envelope, and includes consecutive ring-like structures, termed M (membrane)- S (supramembrane)- L (lipopolysaccharide)- $\mathrm{P}$ (peptidoglycan)- and C (cytoplasm)-rings; the hook is a bended structure that protrudes from the basal body on the cell surface; and the filament is a long (up to several micrometer) tubular structure of $>20,000$ s copies of a singular protein, the flagellin (Nakamura and Minamino, 2019).

The M- and S-rings are formed by the protein FliF, a $\sim 60 \mathrm{kDa}$ protein, embedded in the cytoplasmic membrane through two trans-membrane helices (Figure 1A). It oligomerizes into a circular membrane-spanning complex, forming the fundamental scaffold for flagellar structure and assembly (Minamino et al., 2008). In-between the two transmembrane helices, FliF possesses a large periplasmic region consisting of three globular domains termed Ring-Building Domains (RBM1, RBM2, and RBM3, respectively; Figure 1A; Bergeron, 2016). Those RBMs possess a common fold (Spreter et al., 2009), and show structural homology with components of the Type III Secretion System (T3SS) injectisome, and in particular RBM1 and RBM2 have sequence similarity with the T3SS protein SctJ (Yip et al., 2005; Bergeron et al., 2015; Bergeron, 2016). Conversely, RBM3 shows homology to the SpoIIIAG protein (Bergeron, 2016;
Zeytuni et al., 2017), a macromolecular complex involved in spore formation.

On the cytosolic side, FliF binds to the protein FliG, part of the C-ring (Kubori et al., 1992; Levenson et al., 2012), via its C-terminus. This interaction is necessary for flagellum assembly (Li and Sourjik, 2011; Morimoto et al., 2014). FliG, together with FliM and FliN, form the C-ring, and are responsible for switching of the rotation between clockwise and counterclockwise (Morimoto and Minamino, 2014; Minamino and Imada, 2015).

The assembly of the flagellar motor has been mainly investigated in the model systems Escherichia coli and Salmonella enterica serovar Typhimurium. In these peritrichously flagellated bacteria, the initial component to form is the MS-ring, followed by the C-, P-, and L-rings. A T3SS-like export apparatus is recruited by interaction with the MS-ring and is responsible for secretion of the single components of the rod, hook, and filament, which are then assembled outside the cytoplasm (Minamino et al., 2008; Minamino and Imada, 2015; Nakamura and Minamino, 2019).

While the MS-ring formation occurs early during flagellar biogenesis, it remains unclear which factors are needed for its recruitment and assembly. In E. coli, FliF was not able to efficiently self-oligomerize and assemble at the membrane when expressed at its physiological levels. Instead, it was suggested that the basal body assembly in E. coli is a cooperative process where FlhA firstly assembles to the membrane and subsequently recruits FliF through direct interaction, further aided by FliG and both FlhA and FliG (Li and Sourjik, 2011). In contrast, in S. Typhimurium it was observed that FliF overexpression led to spontaneous assembly of MS-ring structures (Suzuki et al., 2004; Kawamoto and Namba, 2017; Kawamoto et al., 2020; Johnson et al., 2021) whereas in Vibrio alginolyticus the same behavior was not observed (Terashima et al., 2020). Furthermore, co-expression of FlhF and FliG promotes formation of MS-rings in V. alginolyticus (Terashima et al., 2020). These findings are in agreement with previous studies where it was highlighted that FlhF and FlhG are involved

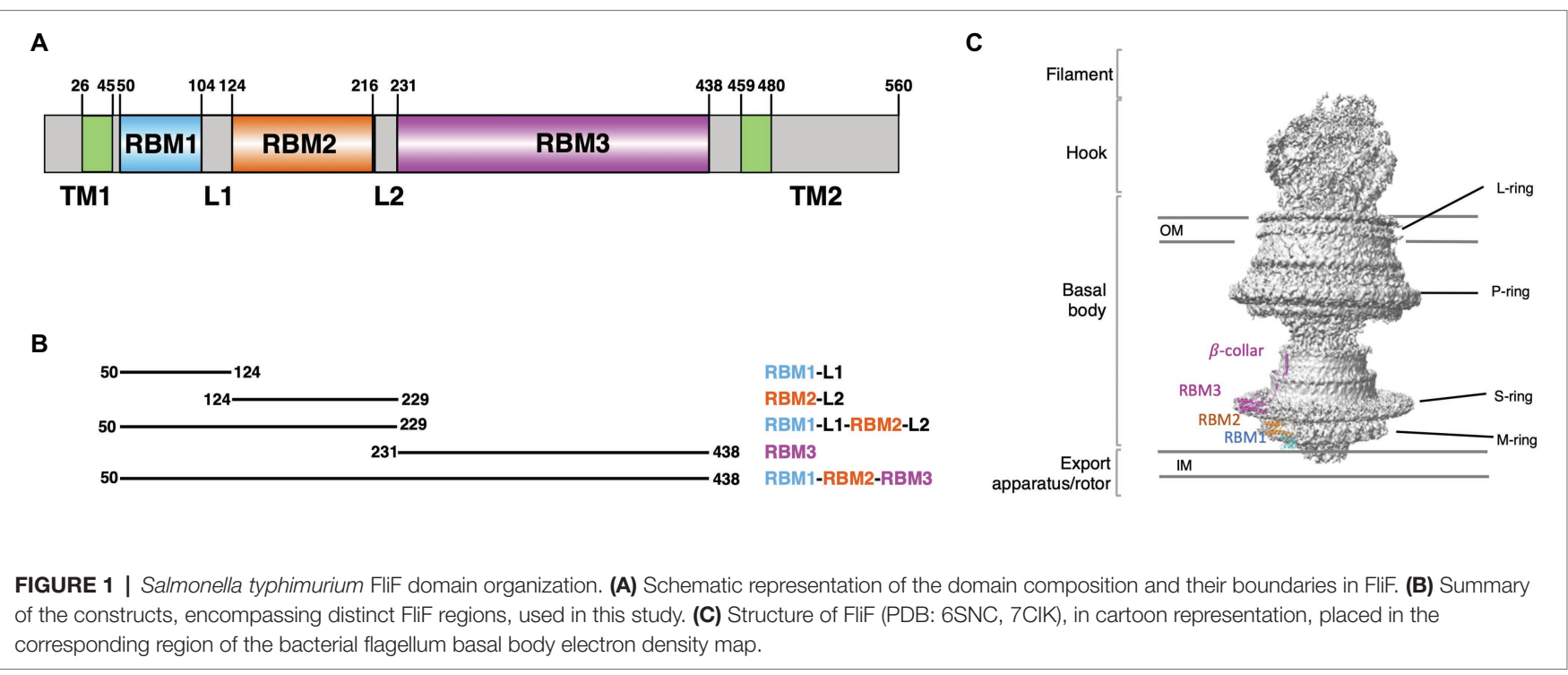


in regulation of flagellar localization and assembly in species with polar flagella and in some peritrichous species such as Bacillus subtilis (Kazmierczak and Hendrixson, 2013). FlhG is a MinD-like ATPase, and interacts with components of the C-ring, FliM, FliN, and FliY (Schuhmacher et al., 2015a,b). Upon interaction with these proteins, FlhG promotes their interaction and assembly with FliG (Schuhmacher et al., 2015a,b). FlhF is a SRP-type GTPase that localizes at the cell pole to positively regulate the localization and formation of the flagellum by recruiting FliF (Green et al., 2009; Terashima et al., 2020), whereas FlhG acts as a negative regulator of flagellar assembly through interaction with FlhF (Kusumoto et al., 2008; Kojima et al., 2020).

The structure of FliF in isolation was recently determined, and revealed that the RBM3 has a symmetry that can vary from C32 to C35, with the majority of particles displaying a C33 symmetry (Johnson et al., 2020). Astonishingly, this study showed that RBM2 forms rings with a 21 or 22 -fold symmetry, localized at the inner part of the M-ring (Johnson et al., 2020), revealing a symmetry mismatch between the domains. RBM1 was not resolved in these structures. Subsequently, it was shown that the prevalent symmetry for the basal body is C34 and that the RBM2 adopts preferentially a C23 symmetry at the internal face of the M-ring (Kawamoto et al., 2020). The cryo-EM structure of the intact basal body further confirmed that the RBM3 unambiguously displays a C34 symmetry (Kawamoto et al., 2020; Johnson et al., 2021). Nonetheless, these structures raise the question of how this protein can form an oligomeric assembly with different symmetries in different domains, and how their assembly is coordinated.

Here, we studied the oligomerization of the different FliF domains in isolation. We show that a construct encompassing RBM1, RMB2, and RBM3, but lacking the two trans-membrane helices, is still able to form the proper MS ring assembly, in the S. Typhimurium ortholog (but not the Helicobacter one). We demonstrate that the RBM2 and RBM3 domains oligomerize in isolation, and form ring-like structures, with symmetry corresponding to that of these domains within the basal body. In contrast, RBM1 in isolation is strictly monomeric. Intriguingly, we also report that a construct encompassing both RBM1 and RBM2 is monomeric, suggesting that within this construct, RBM1 prevents RBM2's oligomerization. Finally, ectopic addition of RBM3 promotes the oligomerization of the RBM1-RBM2 construct, reversing the inhibition of RBM2 oligomerization by RBM1. Taken together, these results suggest that the oligomerization of FliF is coordinated and allows us to propose a model for the regulated formation of the MS ring to the final, correct assembly.

\section{MATERIALS AND METHODS}

\section{Protein Expression and Purification}

The gene coding for FliF encompassing RBM1, RBM2, and RBM3 ( FliF $_{50-438}$ ) was synthesized (Bio Basic), and cloned into the pET-28a vector, to include with a Thrombin-cleavable $\mathrm{N}$-terminal $\mathrm{His}_{6}$ tag. Other FliF constructs (see Figure 1B) were generated by site-directed mutagenesis, using the aforementioned construct as a template.
For protein over-expression, the corresponding plasmids were transformed into E. coli BL21 DE3 cells and grown at $25^{\circ} \mathrm{C}$ at $160 \mathrm{rpm}$ overnight in ZYM-5052 auto-induction media (1\% Tryptone, $0.5 \%$ Yeast Extract, $25 \mathrm{mM} \mathrm{Na}_{2} \mathrm{HPO}_{4}, 25 \mathrm{mM} \mathrm{KH}_{2} \mathrm{PO}_{4}$, $50 \mathrm{mM} \mathrm{NH} \mathrm{NH}_{4} \mathrm{Cl}, 5 \mathrm{mM} \mathrm{Na} \mathrm{SO}_{4}, 2 \mathrm{mM} \mathrm{MgSO}, 0.5 \%$ glycerol, $0.05 \%$ glucose, and $0.2 \% \alpha$-lactose) for $16 \mathrm{~h}$. Following induction, cells were centrifuged at $5,000 \times g$ and pellets resuspended in buffer A containing $50 \mathrm{mM}$ Hepes $\mathrm{pH} 8.0,500 \mathrm{mM} \mathrm{NaCl}$, and $20 \mathrm{mM}$ imidazole. Cells were lysed by sonication following addition of cOmplete ${ }^{\mathrm{TM}}$ EDTA-free protease inhibitor (Sigma) and debris removed by centrifugation at $14,000 \times g$ for $45 \mathrm{~min}$. The cleared supernatant was applied onto a $5 \mathrm{ml}$ HisPure $^{\mathrm{TM}}$ Ni-NTA resin (Thermo Scientific) gravity-based column equilibrated with 10 column volumes of buffer A. Proteins were eluted with a two step-gradient elution containing 50 and $500 \mathrm{mM}$ imidazole, respectively. Fractions containing purified FliF RBM2 ( FliF $_{124-229}$ ) were further purified by size exclusion chromatography (SEC) using a Superdex 200 10/300 column (GE Healthcare) in a buffer containing $50 \mathrm{mM}$ Hepes $\mathrm{pH} 9.0$ and $500 \mathrm{mM} \mathrm{NaCl}$. Purified FliF RBM1-RBM2 ( FliF $\left._{50-229}\right)$ and RBM3 $\left(\right.$ FliF $\left._{231-438}\right)$ were applied to a Superdex 200 10/300 column and to a Superose $610 / 300$ column (GE Healthcare), respectively, in a buffer containing $50 \mathrm{mM}$ Hepes $\mathrm{pH} 8.0$ and $500 \mathrm{mM} \mathrm{NaCl}$.

\section{SEC-MALS Analysis}

Samples were run through a standard bore, $5 \mu 300 \AA$ SEC column (Wyatt), using an infinityII HPLC (Agilent), in buffer containing $20 \mathrm{mM}$ HEPES pH 7.0, $150 \mathrm{mM} \mathrm{NaCl}$, and $1 \mathrm{mM}$ DTT. MALS and DRI data were obtained using the DAWN and Optilab detectors, respectively (Wyatt), and analyzed with the Dynamics software (Wyatt) to determine the molecular mass.

\section{Negative-Stain Grid Preparation and EM Data Acquisition}

For negative-stain EM experiments, $5 \mu \mathrm{l}$ of purified protein, at a concentration of $0.2 \mathrm{mg} / \mathrm{ml}$ were applied onto glow-discharged carbon-coated copper grids, and incubated at $20^{\circ} \mathrm{C}$ for $2 \mathrm{~min}$. The grids were then washed in deionized water, and incubated with $1 \%$ Uranyl Formate for $30 \mathrm{~s}$. For the titration experiments, FliF RBM1-RBM2 (FliF $\left.{ }_{50-229}\right)$ and RBM3 ( FliF $\left._{231-438}\right)$ were mixed at $1: 1$ ratio; and $\mathrm{RBM} 2\left(\mathrm{FliF}_{124-229}\right)$ was kept at a constant concentration of $0.2 \mathrm{mg} / \mathrm{ml}$, while RBM1 $\left(\mathrm{FliF}_{50-124}\right)$ was added at different ratios, respectively.

Images were acquired on a Technai T12 Spirit TEM (Thermo Fisher) equipped with an Orius SC-1000 camera (Gatan). For FliF RBM2 $\left(\right.$ FliF $\left._{124-229}\right)$ domain, images were acquired at a $49 \mathrm{k}$ magnification with a defocus range of -0.5 to $-1.0 \mu \mathrm{m}$. For FliF RBM3 $\left(\mathrm{FliF}_{231-438}\right)$ domain, images were acquired at a $30 \mathrm{k}$ magnification with a defocus range of -0.5 to $-1.0 \mu \mathrm{m}$.

\section{Cryo-EM Grid Preparation, Data Collection, and Data Processing}

About $5 \mu \mathrm{l}$ of protein at a concentration of $10 \mathrm{mg} / \mathrm{ml}$, in $50 \mathrm{mM}$ Hepes ( $\mathrm{pH}$ 9.0) and $150 \mathrm{mM} \mathrm{NaCl}$, was applied 
onto glow-discharged 300 mesh Quantifoil R1.2/1.3 grids. Grids were then blotted for $10 \mathrm{~s}$ at $80 \%$ humidity, and plunged into liquid ethane, using a Leica EM-GP plunge freezer.

For RBM2 (FliF $\left.F_{124-229}\right)$, micrographs were collected on a $300 \mathrm{kV}$ Titan Krios microscope equipped with a Gatan K3 camera. 10,053 movies were recorded with a pixel size of $0.85 \AA$ and an exposure of $1 \mathrm{e}^{-} / \AA^{2} /$ frame for $40-50$ frames. For RBM1-RBM2-RBM3 $\left(\mathrm{FliF}_{50-438}\right)$, micrographs were collected on a $200 \mathrm{kV}$ Tecnai Arctica equipped with a Falcon 3 camera. A total of 2,540 movies were collected using a pixel size of $2.03 \AA$ and an exposure of $0.8 \mathrm{e}^{-} / \AA^{2} /$ frame over 50 frames.

Data processing was performed in RELION 3.1 (Scheres, 2020). Motion correction was performed with MotionCor2 (Zheng et al., 2017). CTF parameters were estimated with CTFFIND4 (Rohou and Grigorieff, 2015). For RBM2 (FliF ${ }_{124-229}$ ), 2,000 micrographs were manually picked and used for training a model for particle picking in crYOLO 1.5 (Wagner et al., 2019). The trained model was then used for automated particle picking for the whole dataset and box files were imported on RELION 3.1 for particle extraction. A total of $\sim 2,000,000$ particles were extracted with a 230 pixels box. Extracted particle were subjected to multiple rounds of $2 \mathrm{D}$ classification to filter top views that allowed evaluation of symmetry. For RBM1-RBM2-RBM3 ( FliF $\left._{50-438}\right)$, automated picking was instead performed within RELION 3.1, and a total of 129.000 particles were extracted with a box size of 220 pixels.

\section{Sequence Analysis and Model Docking}

The co-evolution analysis between RBM1 $\left(\mathrm{FliF}_{50-124}\right)$, and RBM2 $\left(\right.$ FliF $\left._{124-229}\right)$ was performed with the RaptorX Complex Contact prediction server (Zeng et al., 2018), using default parameters. To model the interaction between RBM1 ( FliF $\left._{50-124}\right)$, and RBM2 $\left(\right.$ FliF $\left._{124-229}\right)$ based on the co-evolution data, we first generated a homology model of the $S$. Typhimurium RBM1, based on the A. aeolicus RBM1-RBM2 crystal structure (PDB ID: 7CIK, Figure 1C). We then employed the HADDOCK 2.4 server to predict the structure of a complex formed between this homology model and the RBM2 structure (from PDB ID 6SD4), with all the co-evolving residues with a score above 0.4 included as active residues in the interaction. About 200 decoys were modeled, which could be classified in 10 clusters, three of which were very similar, with identical interaction interfaces and RMSD $<4 \mathrm{~A}$. These included the lowest-energy model, and combined represented 55 decoys, suggesting that it is likely close to the real complex structure.

\section{RESULTS}

\section{Oligomerization of Individual Domains of FliF}

Previous studies had shown that when purified in isolation, the $S$. Typhimurium FliF adopted its oligomeric state, including an unusual symmetry mismatch between RBM2 and RBM3 (Johnson et al., 2020; Kawamoto et al., 2020), suggesting a complex folding and assembly pathway for the MS ring. This observation prompted us to investigate if the individual RBMs could oligomerize on their own.

To this end, we engineered a series of constructs that encompassed one or several RBMs (Figure 1B; Table 1). For each construct, the correspondent protein was purified, and its oligomerization propensity was analyzed by SEC (Table 1; Figure 2A; Supplementary Figure S1).

As shown on Figure 2A, a construct encompassing RBM1, RBM2, and RBM3 ( $\left.\mathrm{FliF}_{50-438}\right)$ forms a high-order oligomer, stable by SEC. Negative-stain EM (ns-EM) analysis revealed that the protein possessed ring-like features (Figure 2C), similar to that of the full-length protein. This demonstrates that the TM helices of FliF are dispensable for its oligomeric assembly. These findings are perhaps not surprising, considering that in some bacterial species, the T3SS equivalent of FliF, SctJ, lacks a N-terminal TM helix (Crepin et al., 2005; Yip et al., 2005). Nonetheless, SctJ also possesses a C-terminal palmytoilation site, not present in FliF, and required for membrane localization and assembly.

We however note that the protein is prone to aggregation, with multiple MS rings assembling from the side opposing the collar region, suggesting that some hydrophobic surfaces, possibly facing the membrane, are exposed in the absence of the TM helices. Indeed, SEC-MALS analysis confirmed that FliF from $S$. Typhimurium $\left(\mathrm{StFliF}_{50-438}\right)$ self-oligomerized in a complex with an apparent mass of $10 \mathrm{MDa}$ (Supplementary Figure S2A), significantly larger than the FliF 34-mer. This is consistent with the ns-TEM experiment reported above (Figure 2C), which showed that most of the StFliF ${ }_{50-438}$ oligomers were sticking together.

Next, we observed that constructs encompassing RBM2 $\left(\right.$ FliF $\left._{124-229}\right)$ or RBM3 $\left(\mathrm{FliF}_{231-438}\right)$ also formed higher-order

TABLE 1 | List of S. Typhimurium constructs used in this study, with their corresponding predicted MW, and that calculated by their SEC elution volume.

\begin{tabular}{|c|c|c|c|c|c|c|}
\hline Domain & $\begin{array}{c}\text { Construct } \\
\text { boundaries }\end{array}$ & $\begin{array}{l}\text { Monomer MW } \\
\text { (KDa) }\end{array}$ & $\begin{array}{l}\text { Oligomeric state of } \\
\text { the purified protein }\end{array}$ & $\begin{array}{l}\text { Elution volume } \\
\qquad(\mathrm{Ve}, \mathrm{ml})\end{array}$ & $\begin{array}{c}\text { Apparent (by SEC) } \\
\text { molecular weight (KDa) }\end{array}$ & $\begin{array}{c}\text { Predicted molecular } \\
\text { weight (KDa) }\end{array}$ \\
\hline RBM1 + L1 & $50-124$ & 8.13 & Monomer & 18.90 & 7.56 & 8.13 \\
\hline $\mathrm{RBM} 2+\mathrm{L} 2$ & $124-229$ & 11.21 & Oligomer & 9.35 & 390.15 & 257.83 \\
\hline $\mathrm{RBM} 1+\mathrm{L} 1+\mathrm{RBM} 2+\mathrm{L} 2$ & 50-229 & 19.25 & Monomer & 17.65 & 11.93 & 19.25 \\
\hline RBM3 & $231-438$ & 22.50 & Oligomer & 8.66 & 868.80 & 765.00 \\
\hline $\mathrm{RBM} 1+\mathrm{RBM} 2+\mathrm{RBM} 3$ & $50-438$ & 41.87 & Oligomer & 8.27 & 1046.44 & 1422.56 \\
\hline Full length & $1-560$ & 63.95 & - & - & - & - \\
\hline
\end{tabular}


A

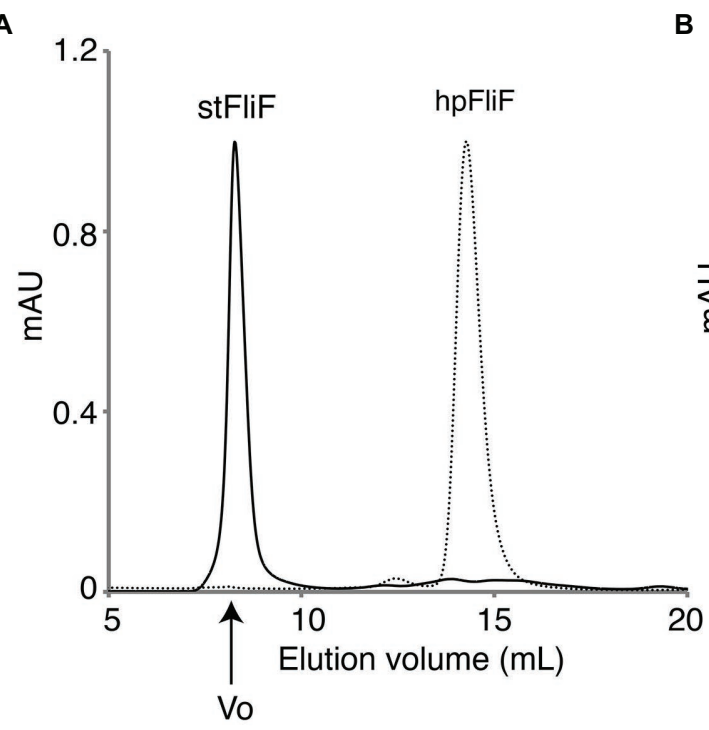

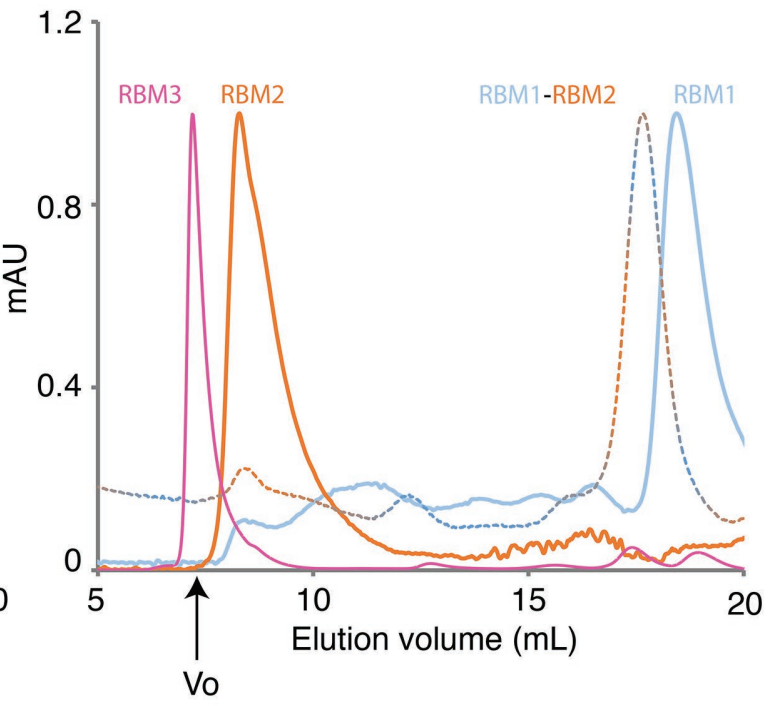

C

FliF50-438 (RBM1-RBM2-RBM3)

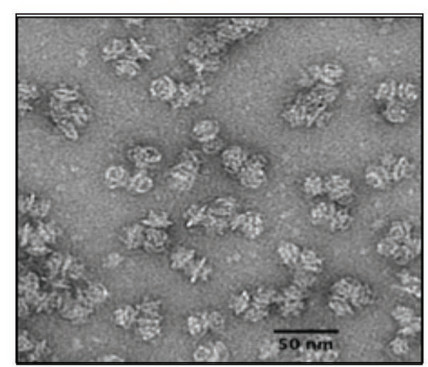

D

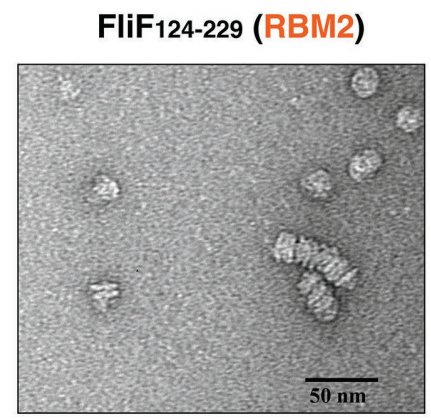

E FliF231-438 (RBM3)

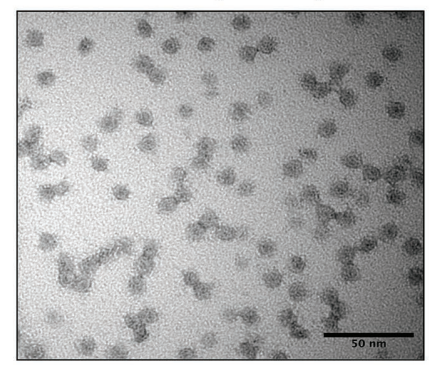

FIGURE 2 | Oligomerization of the FliF domains. (A) Size exclusion chromatography (SEC) UV trace of constructs encompassing the entire periplasmic regions of S. typhimurium FliF and Helicobacter pylori FliF. (B) SEC UV trace of constructs encompassing the individual domains of S. typhimurium FliF (C-E) Negative stain analysis of (C) RBM1-RBM2-RBM3 (FliF $50-438$ ), (D) RBM2 (FliF ${ }_{124-229}$ ), and (E) RBM3 (FliF $231-438$ ). RBM1-RBM2-RBM3 and RBM2 show mostly side views, while RBM3 mainly displays top views.

oligomers in isolation (Figure 2B). Negative-stain EM analysis confirmed that they adopted ring-like structures (Figures 2D,E), consistent with their architecture within the native MS ring. In the instance of RBM2 ( FliF $_{124-229}$ ), we note that the ring-like structures exhibited a tendency to cluster together, forming lines of disks (Figure 2D). It is noteworthy that in the T3SS FliF homolog SctJ, previous biochemical studies have shown that RBM2 is monomeric, and requires the $\mathrm{L} 1$ linker to oligomerize in isolation (Bergeron et al., 2015).

In contrast to RBM2 (FliF $\left.{ }_{124-229}\right)$ and RBM3 (FliF $\left.F_{231-438}\right)$, we observed that the construct encompassing RBM1 ( FliF $\left._{50-124}\right)$ was strictly monomeric in isolation (Figure 2B; Table 1). Collectively, these results demonstrate that in S. Typhimurium, the TM helices of FliF are dispensable for its oligomeric assembly, and that RBM2 and RBM3, but not RBM1, can form oligomeric rings in isolation.
While we observed that RBM1-RBM2-RBM3 (FliF $\left.F_{50-438}\right)$ in $S$. Typhimurium spontaneously oligomerized, previous studies have shown that in other non-peritrichous organisms, such as $V$. alginolyticus, FliF required additional flagellum components, such as FhlF, to efficiently assemble MS-rings (Terashima et al., 2020). For this reason, we investigated the oligomeric state of FliF in another non-peritrichous organism, Helicobacter pylori. We observed that $H$. pylori RBM1-RBM2-RBM3 ( $\left.\mathrm{HpFliF}_{51-427}\right)$ eluted from the gel filtration column much later than $\mathrm{StFliF}_{50-438}$, consistent with a monomeric protein (Figure 2A). SEC-MALS analysis was used to measure the molecular weight of this purified protein (Supplementary Figure S2B), which was measured to be $\sim 41 \mathrm{kDa}$, very close to the predicted molecular weight of a single monomer $(42 \mathrm{kDa})$. This result suggests that in $H$. pylori, FliF requires additional factors to trigger oligomerization, as also reported in other non-peritrichous organisms (Dasgupta et al., 2003; Hendrixson and DiRita, 2003). 


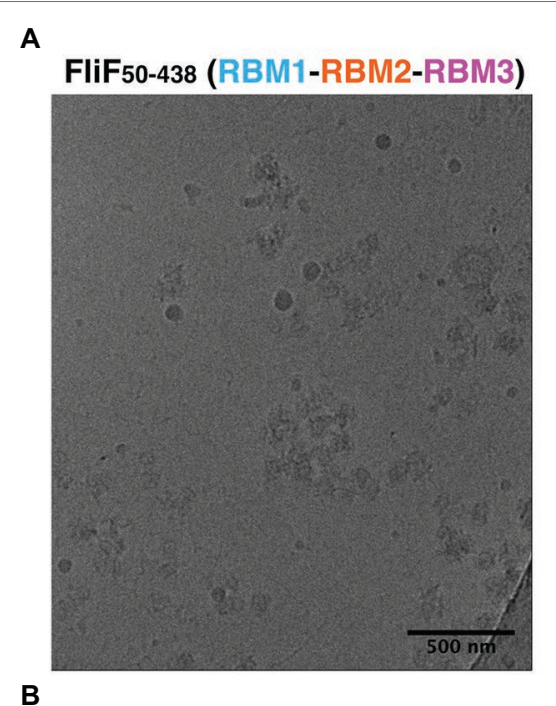

B

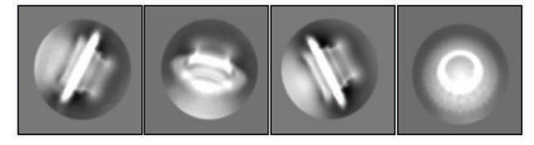

c

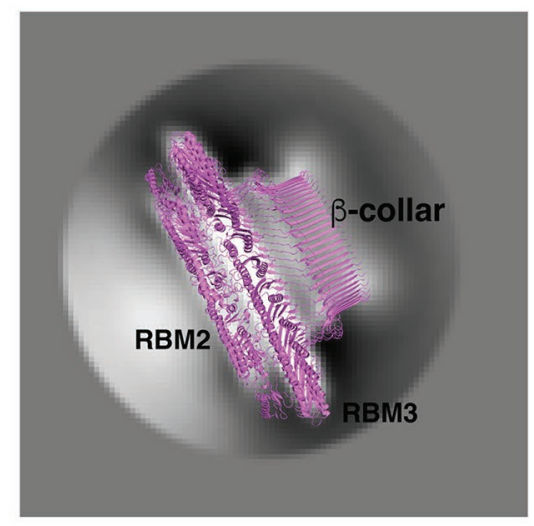

D

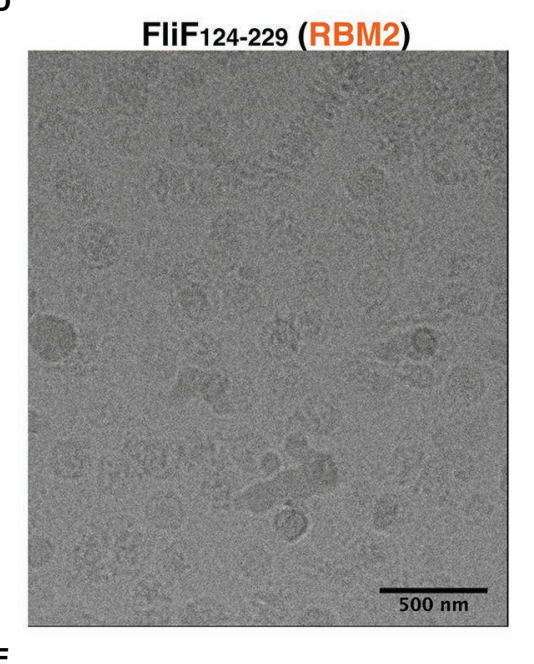

E

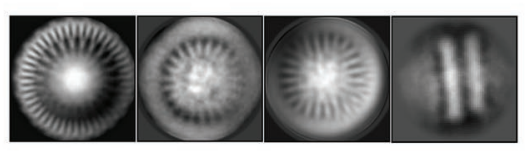

F

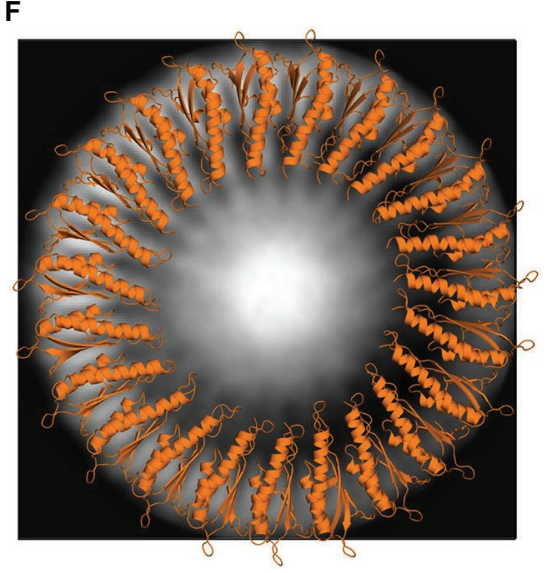

FIGURE 3 | FliF RBM2 and RBM3 stoichiometry. (A) Cryo-electron micrograph and (B) selected 2D classes of RBM1-RBM2-RBM3 (FliF $50-438)$. (C) A 2D class from a side view of RBM1-RBM2-RBM3 is shown, overlayed to a cartoon representation of the 33-mer FliF structure (PDBID: 6SD1). The majority of particles is side view, and matches the architecture of intact FliF. (D) Cryo-electron micrograph, and (E) selected 2D classes of RBM2 (FliF ${ }_{124-229}$ ). Particles show top views and some filaments that consist of aggregation of single RBM2 disks. (F) A 2D class of a RBM2 top view is shown, overlayed to a cartoon model of the $23-$ mer RBM2 inner ring structure (from PDBID: 7BKO).

\section{Cryo-EM Analysis of the FliF RBM2 and RBM3}

The structures of FliF revealed a range of stoichiometries, from 32 to 34 for RBM3, and 21 or 22 for RBM2, with an extra 11-12 RBM2 domains in a distinct orientation relative to RBM3, and facing outward (Johnson et al., 2020). Subsequent structures of this protein in the intact basal body demonstrated that the true stoichiometries are 34 and 23, respectively (Kawamoto et al., 2020; Johnson et al., 2021). This prompted us to use cryo-EM to characterize the oligomeric constructs described above, to confirm that they match the structure of the native FliF oligomer, and determine the stoichiometry of the individual domains.
As shown on Figure $\mathbf{3 A}$, the FliF construct encompassing RBM1, RBM2, and RBM3 ( FliF $\left._{50-438}\right)$ was readily incorporated into ice, which allowed us to collect a cryo-EM dataset. Because of the high level of aggregation (see above), we picked particles from this data manually, and used these to generate $2 \mathrm{D}$ classes (Figure 3B). These 2D classes are highly similar to that of the MS ring in isolation, with density for RBM2, RBM3, and the $\boldsymbol{\beta}$-collar clearly visible (Figure $3 \mathrm{C}$ ). Diffuse density below RBM2 is also visible, and was also seen in previously-reported $2 \mathrm{D}$ classes of the full MS ring, corresponding to density for dynamic RBM1 domains.

While most particles were attributed to $2 \mathrm{D}$ classes corresponding to side-views of the complex, a subset $(\sim 10 \%)$ 
corresponded to top views (Figure 3B, far right). Notably, in this class, we were able to clearly identify a 33-fold symmetry (Supplementary Figure S3A). This is in agreement with the structure of FliF in isolation, reported previously (Johnson et al., 2020), where RBM3 adopts a 33-mer stoichiometry in the majority of particles. Further work will be required to determine if our construct also adopts a range of stoichiometries.

Next, we used cryo-EM to characterize the RBM2 ( FliF $\left._{124-229}\right)$ oligomer. This protein was also readily incorporated into ice (Figure 3D), and we were able to collect a cryo-EM dataset. We attempted automated particle picking using a range of tools, but only cryOLO (Wagner et al., 2019) was able to pick both side and top views, in particular as the side views consisted of long aggregation of disks (see above). Using these particles, we generated 2D classes in Relion (Scheres, 2012; Figure 3E). These confirmed that this protein had a pathological level of preferred orientation, with most particles visible from the top of the ring, and very few tilted or side views, with the side views clustered together, as seen in negative stain (see above). This precluded high-resolution structure determination but allowed us to exploit the top views to infer the symmetry of the particles.

In the intact FliF structure in isolation, RBM2 forms two rings: one inner ring with 21 subunits, and one outer ring with nine subunits. As shown on Figure 3F, we can observe on these 2D classes clear density for the two helices of RBM2, notably with a 23-fold symmetry (Supplementary Figure S3B). Additional classification, using a larger top-view dataset would be required if this sample is heterogeneous and includes a range of symmetries, as observed for the intact FliF. Nonetheless, this demonstrates that the oligomers obtained for our RBM2 construct $\left(\mathrm{FliF}_{124-229}\right)$ correspond to the inner ring alone, and does not include the outer ring.

Finally, we note that in the RBM2 ( FliF $\left._{124-229}\right) 2 \mathrm{D}$ classes, some density is visible in the center of the ring, which cannot be interpreted with the current structures of FliF. We propose that this density likely corresponds to some undetermined chemical that was co-purified with the protein. Further work will be necessary to determine the nature of this additional density.

Collectively, these observations confirm that the FliF transmembrane helices are not required for it to adopt its native MS-ring architecture. In addition, we show that both the RBM2 and RBM3 of FliF adopt their native oligomeric conformation in isolation.

\section{RBM1 Prevents the Oligomerization of RBM2, and This Effect Is Counteracted by RBM3}

Previous work on the T3SS FliF homologue SctJ had shown that RBM2 self-oligomerizes, similarly to FliF, but that this oligomerization is repressed in the presence of RBM1 (Bergeron et al., 2015, 2018). We therefore sought to verify if the RBM1 of FliF played a similar role. To that end, we engineered a FliF construct that encompassed both $\mathrm{RBM} 1$ and $\mathrm{RBM} 2\left(\mathrm{FliF}_{50-229}\right)$. As shown on Figure 2A, SEC analysis demonstrated that the resulting protein was strictly monomeric (Figure 1B; Table 1). This suggests that RBM1 prevents RBM2 from oligomerizing on its own.
In order to determine how RBM1 could inhibit RBM2 domains to oligomerize, we first performed co-evolution analysis to determine amino-acid residues that were potentially involved into the interaction between RBM1 and RBM2, using RaptorX Complex Contact prediction server (Zeng et al., 2018). As shown in Supplementary Figure S4A, several regions of the protein, largely corresponding to the $\beta$-strands, showed significant co-evolution scores. Next, we employed the HADDOCK docking server to model the interaction between the two domains, using these residues as restraints in the docking process. This led to a cluster of models with low energy score, where the two domains had their $\beta$-sheet facing each other (Supplementary Figure S4B), in agreement with the co-evolution analysis. Furthermore, overlay of this model onto the RBM2 23-mer structure had RBM1 in the position of an adjacent RBM2 molecule (Supplementary Figure S4C), providing a potential explanation of how the intramolecular contacts between RBM1 and RBM2 sterically obstruct the RBM2 oligomerization. This is consistent with our observation that the RBM2 oligomerization is inhibited by RBM1.

This effect mentioned above was observed in the context on a RBM1-RBM2 construct. This led to the question of whether the addition of ectopic RBM1 (FliF $\left.5_{50-124}\right)$ onto assembled RBM2 ( FliF $\left._{124-229}\right)$ rings promoted their dissociation. To verify this, we titrated purified $\mathrm{RBM1}\left(\mathrm{FliF}_{50-124}\right)$ against oligomeric RBM2 ( FliF $_{124-229}$ ) and used ns-EM to investigate if the ectopic addition of RBM1 disrupted the RBM2 oligomers (see above). As shown on Supplementary Figure S5, we observed no changes in the architecture or density of the RBM2 oligomers, even in large excess of RBM1. This observation demonstrates that once the RBM2 ring is formed, it can no longer be disrupted by RBM1, and suggests that in the context of the RBM1-RBM2 ( FliF $\left._{50-229}\right)$ construct, RBM1 prevents RBM2 oligomerization by binding to the ring oligomerization interface.

Given that RBM1-RBM2 ( FliF $\left._{50-229}\right)$ was shown to be strictly monomeric, while RBM1-RBM2-RBM3 $\left(\mathrm{FliF}_{50-438}\right)$ assembled into the MS ring (Figure 2; Table 1), we further investigated whether addition to RBM3 $\left(\mathrm{FliF}_{231-438}\right)$ would prompt RBM1RBM2 ( FliF $_{50-229}$ ) to oligomerize. To this end, purified RBM1RBM2 ( FliF $\left._{50-229}\right)$ and RBM3 ( FliF $\left._{231-438}\right)$ were mixed (Figure 4A), and ns-EM was employed to test the formation of the intact MS ring. Surprisingly, while we observed presence of ring-like structures formed by RBM3 $\left(\mathrm{FliF}_{231-438}\right)$ alone, we also observed the presence of long tubular structures (Figure 4B). These are distinct in appearance from the lines of disks observed for our RBM2 construct (see Figure 2C), but also to the RBM1RBM2-RBM3 oligomers (See Figure 2B). These tubular structures are reminiscent in the supercoil arrangement observed in the crystal structure of the E. coli SctJ orthologue (Yip et al., 2005). Indeed, projection of the crystallographic symmetry-generated SctJ supercoiled structure looked strikingly similar to the tubular structures we observed for our RBM1-RBM2 construct in the presence of RBM3 added ectopically (Figure 4B; Supplementary Figure S6). We therefore propose that these tubular structures correspond to RBM1-RBM2 oligomers, in a supercoiled arrangement, and are likely capped by RBM3. This interpretation would however require to be experimentally 
verified. Nonetheless, this observation suggests that while RBM1RBM2 exists as a monomer, addition of RBM3 is the determinant factor that pushes toward assembly of FliF into an oligomeric state.

Collectively, these results suggest an intricate set of interactions between the different FliF domains; with RBM1 binding to RBM2 to prevent its oligomerization, and RBM3 acting to prevent this interaction.

\section{DISCUSSION}

The MS-ring assembly is one of the first steps that occur during biogenesis of the flagellum (Minamino et al., 2008). The MS-ring then functions as a scaffold to recruit the C-ring through the interaction of FliF with FliG (Li and Sourjik, 2011; Morimoto et al., 2014) and the export apparatus (Minamino et al., 2008; Minamino and Imada, 2015; Nakamura and Minamino, 2019). Despite this central role in flagellum assembly, the process and regulation underlying the MS-ring folding remain unknown. A deeper understanding of the FliF folding process has become increasingly important in light of the recent structural studies that have reported the existence of distinct symmetries within the MS-ring, which could serve multiple functions (Johnson et al., 2020, 2021; Kawamoto et al., 2020; Takekawa et al., 2021).

Indeed recent structural analyses have highlighted that the MS-ring symmetry can adopt a range of oligomeric states, with a mismatched symmetry between RBM2 and RBM3 (Johnson et al., 2020). While initially this suggested that RBM3 adopted a range of stoichiometries that range from 32 to 34 subunits, and that RBM2 formed either 21 or 22 -mer (Johnson et al., 2020), in subsequent studies it was consistently observed that RBM3 was a 34-mer and RBM2 was a 23-mer (Kawamoto et al., 2020; Johnson et al., 2021). The symmetry mismatch between RBM2 and RBM3, together with the different symmetries detected in the existing studies suggests the existence of a complex process that regulates the folding and biogenesis of the MS-ring. In this study, we aimed to determine the mechanism underlining the complex folding of FliF, by analyzing the oligomeric state of the different domains of FliF.

Here, we show that in a construct encompassing FliF RBM1, RBM2, and RBM3 is able to assemble to form MS-rings, wherein RBM3 displays a 33-mer stoichiometry. Additionally, our data reveal that RBM2 is able to form rings with a 23-mer stoichiometry. These correspond to the main stoichiometry observed for FliF in isolation. Conversely, we observe that a construct encompassing RBM1-RBM2-RBM3 $\left(\mathrm{HpFliF}_{51-427}\right)$ from H. pylori yields a monomeric protein. These findings show similarities with what was observed for FliF in $V$. alginolyticus, where its proper oligomerization required additional factors and suggest the existence of a different regulation of the MS-ring assembly for non-peritrichous organisms (Terashima et al., 2020).

Indeed, our data demonstrate that a construct encompassing RBM1 and RBM2 is monomeric, contrary to a construct encompassing RBM2 only. Since the addition of RBM1 to already formed RBM2 rings does not show any changes, we propose
A
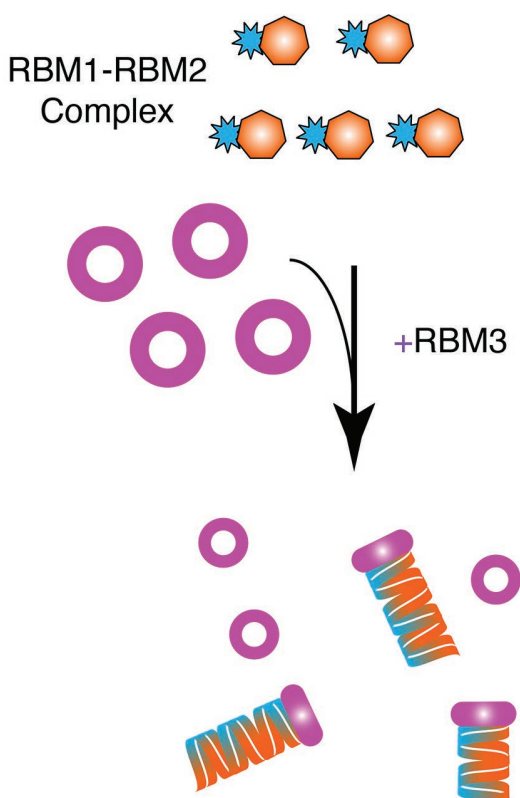

B

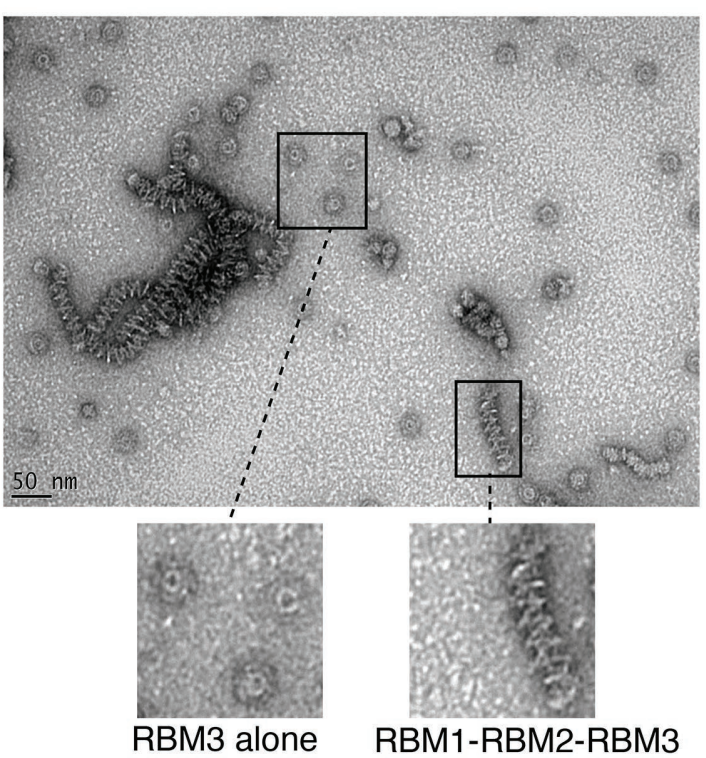

FIGURE 4 | Ring-Building Motif (RBM)3 induces oligomerization of the RBM1-RBM2 construct. (A) Schematic representation of the experiment. RBM1-RBM2 $\left(\right.$ FliF $\left._{50-229}\right)$ was mixed with RBM3 (FliF $231-438$ ) oligomers and imaged by negative stain EM. (B) Negative stain electron micrograph of the RBM3 and RBM1-RBM2 mixture. Ring-like structures, representative ofRBM3alone, are visible, as well as tubular structures that are likely composed of stacks RBM1-RBM2-RBM3 rings. 
that RBM1 prevents the RMB2 oligomerization by binding to, and thus occluding, its oligomerization interface. Additionally, we also show that addition of RBM3 to monomeric RBM1RBM2 causes formation of tubular structures, which we attribute to RBM1-RBM2 adopting a superhelical fold, similarly to SctJ (Yip et al., 2005), possibly capped by RBM3. This in turn suggests that RBM3 interacts with the RBM1-RBM2 construct in a way that dislodges RBM1 and allows RBM2 to oligomerize.

Based on this, we propose the following mechanism for MS ring assembly: Upon membrane insertion by the SEC pathway, FliF is a monomer; the interaction between RBM1 and the oligomerization interface on RBM2 retains FliF in a monomeric state (Figure 5A). Next, while RBM1 still prevents RBM2 molecules from associating, RBM3 oligomerization initiates (Figure 5B), imposing an overall 34-mer stoichiometry to the complex. Assembled RBM3 rings can subsequently disrupt RBM1 from RBM2 oligomerization interface, and RBM2 rings start forming (Figure 5C). These form 23-mer rings, but because the overall stoichiometry is imposed by the initial RBM3 oligomerization, 11 RBM2 domains are left on the outside. Therefore, we propose that the role of RBM1-mediated inhibition of RBM2 oligomerization in the FliF assembly process allows $\mathrm{RBM} 3$ rings to form and drive the MS-ring biogenesis process, determining the right stoichiometry for all the sub-assemblies. This leads to the formation of the intact MS-ring, with its symmetry mismatch between RBM2 and RBM3 (Figure 5D).

The concept that the three periplasmic domains RBM1RBM2-RBM3 of FliF provide regulation of its oligomerization, thus guaranteeing the right stoichiometry of the MS-ring and the consequent correct assembly of the basal body, is not foreign. Indeed a similar regulation has been proposed in the evolutionarilyrelated T3SS secretion apparatus (Yip et al., 2005; Bergeron et al., 2015, 2018; Bergeron, 2016). There, the model suggests that the SctJ linker between RBM1 and RBM2 interacts with
A

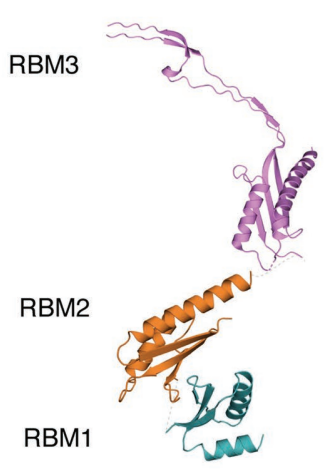

FliF monomer

C

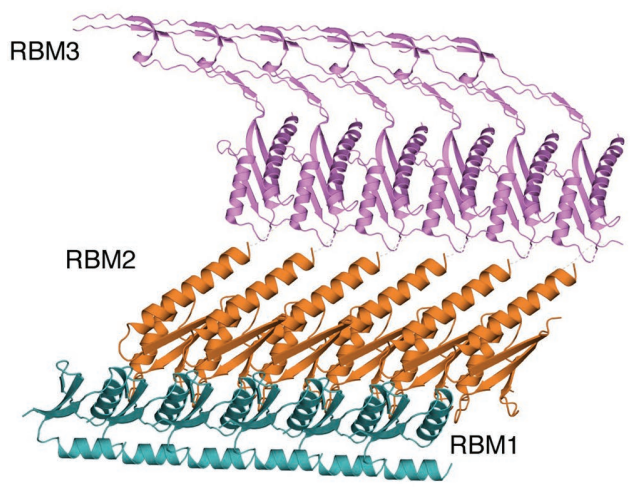

RBM3 oligomerization displaces RBM1 domains and allows RBM2 domains association

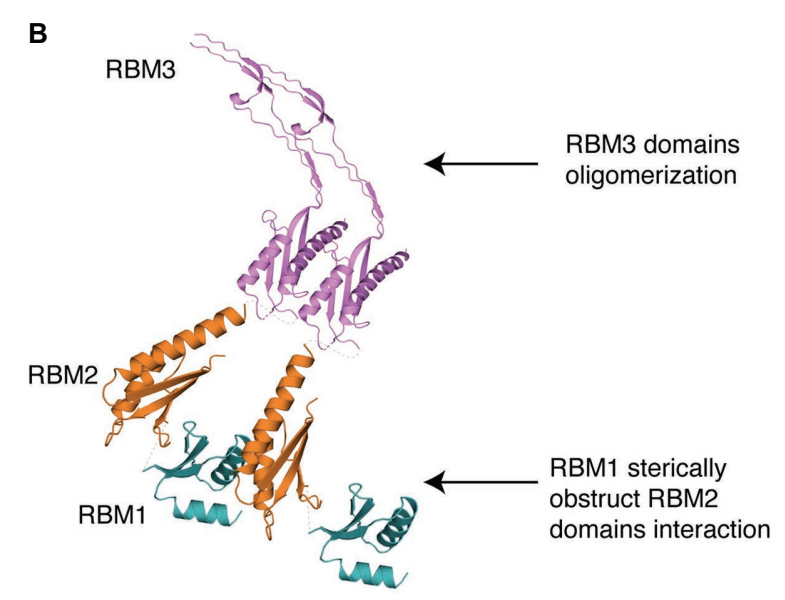

D

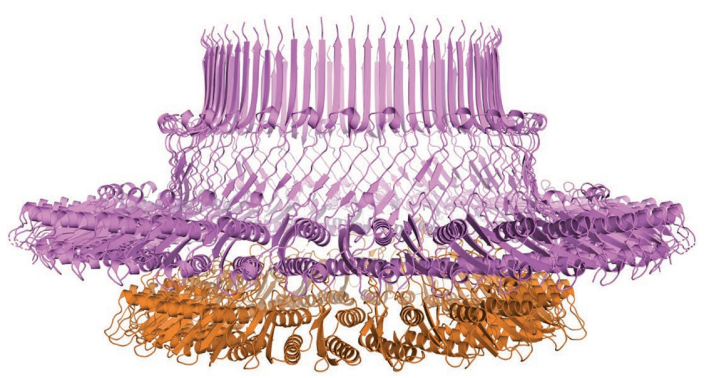

Final MS-ring

FIGURE 5 | Structural representation of the proposed MS-ring assembly process. Cartoon representations of each domain, colored as in Figure 1, are used. (A) As it gets exported to the membrane via the Sec pathway, FliF is monomeric, (B) The presence of adjacent FliF molecules allows RBM3 to drive the oligomerization process, establishing the 34-mer stoichiometry. The contacts between RBM1 and RBM2 prevent RBM2 to oligomerize. (C) At completion of RBM3 oligomerization, RBM1 is displaced from RBM2, allowing RBM2 domains from different FliF molecules to come into contact and assemble, forming a 23-mer. The remaining 11 RBM2 domains sit outside this ring. (D) In the final stage, the MS-ring reaches the final correct conformation, with asymmetry mismatch between RBM2 and RBM3. 
RBM1 with hydrophobic interaction, keeping SctJ in a monomeric state (Bergeron et al., 2015, 2018). Upon dissociation of the linker region from RBM1, SctJ subunits can associate, and establish a series of interactions between their respective RBM1RBM2 domains, as well as with the linker region (Bergeron et al., 2015, 2018). SctD molecules subsequently insert between two adjacent SctJ subunits, and SctJ-SctD heterodimers can finally oligomerize to form the finalized rings (Bergeron et al., 2015, 2018). Our data show that while in FliF, RBM1 and RBM3 can provide regulation of RBM2 oligomerization; it appears that the process is opposite to what observed in SctJ.

The functional implication for this difference is not known; however, it is tempting to speculate that it might be related to a regulatory process, to fine-tune the assembly and/or disassembly of these complexes. Notably, most T3SS complexes are required only in the context of an interaction with target cell membranes, and it is therefore likely to have a disassembly pathway (although this has not been observed directly). In contrast, the bacterial flagellum plays multiple roles in the bacterial cell, beyond motility, including cellular localization, and regulation of cell division (Chaban et al., 2015). Therefore it is likely a much more stable complex, with the basal body constantly anchored at the cell pole in many (but probably not all) bacterial species. The difference observed between the T3SS and flagellar inner-membrane components could reflect these distinct properties. Further investigation, combining biochemical characterization and in vivo assays, in both systems and in a range of bacterial species, would be required to decipher this.

It is worth to note that for SctJ the regulation role was pin-pointed to the linker region, and notably to a conserved phenylalanine residue, necessary for RBM2 oligomerization, and controlled by the isomerization of a proline residue. In this study, the FliF RBM2 construct we used does not encompass the linker between RBM1 and RBM2, and thus, it appears that this linker does not play a critical role in FliF oligomerization. In addition, the aforementioned Phe or Pro residues are not conserved in the corresponding region of FliF (Bergeron, 2016; Bergeron et al,, 2018), further supporting the distinction between FliF and SctJ.

The biogenesis of the flagellum is a hierarchical process that initiated with the insertion of the Type III export apparatus and the assembly of the MS-ring. The remaining flagellar components are then secreted through the export apparatus to build up the final flagellar structure (Yonekura et al., 2002; Macnab, 2003). The levels of regulation of this process are complex, relying on the hierarchical and timely transcription of the distinct components of the flagellum, which are transcribed in different groups according to their role in the flagellar structure (Kutsukake et al., 1990; Dasgupta et al., 2003). In a similar fashion, it is possible to speculate that RBM1 and RBM3-mediated control over the oligomerization and assembly of the MS-ring will provide an additional level of complexity to the flagellum biogenesis.

Several studies have shown that the regulation process involves different factors between peritrichous and polar flagella. Namely, FlhF and FlhG are not present in E. coli and S. Typhimurium but are necessary for flagellar synthesis and localization in a number of species (Pandza et al., 2000; Hendrixson and DiRita, 2003; Niehus et al., 2004; Kusumoto et al., 2008; Schuhmacher et al., 2015a). Interestingly, FlhF and FlhG were found to antagonistically influence the levels of expression of the distinct groups of genes involved in flagellum synthesis (Dasgupta et al., 2003; Hendrixson and DiRita, 2003). It is also noteworthy that in some species carrying FlhF and FlhG, FliF was found to remain in a monomeric state in vitro and oligomerization occurred only in presence of FlhF and FliG (Terashima et al., 2020). In this study, we reported that FliF in $H$. pylori exists in a monomeric state in vitro. In other non-peritrichous organisms, such as V. alginolyticus, FliF requires additional factors to efficiently form MS-rings structures (Terashima et al., 2020). Given the non-peritrichous nature of the flagella of these two organisms, it is possible to speculate that in H. pylori FliF may also require FlhF or other additional factors to trigger its oligomerization, suggesting distinct assembly models between peritrichous and mono/amphitrichous bacteria, which will require further investigation.

Conversely, in S. Typhimurium, it has been shown that FliF can oligomerize spontaneously (Minamino et al., 2008; Johnson et al., 2020). Nevertheless, distinct regulation strategies were also found between peritrichous flagella. Indeed, FhlA was shown to firstly assemble at the membrane of E. coli and to be pivotal for FliF recruitment (Li and Sourjik, 2011).

These observations underline that different, multi-faceted mechanisms of regulations exist for correct assembly of the flagellar machinery between species and that control of FliF oligomerization in $S$. Typhimurium, provided by FliF own domains, adds a new level of complexity to the modulation of the flagellum biogenesis.

Ultimately, characterization of the differences in the assembly of the flagellum between species will provide a better understanding of the molecular elements that determine regulation of the flagellum.

\section{DATA AVAILABILITY STATEMENT}

The original contributions presented in the study are included in the article/Supplementary Material, further inquiries can be directed to the corresponding author.

\section{AUTHOR CONTRIBUTIONS}

RF-R and JR cloned the various constructs. GM, RF-R, SB, $\mathrm{WZ}$, and JR purified the proteins. GM and RF-R performed the negative-stain EM and cryo-EM analyses, and processed the cryo-EM data, with help from SBT. JRCB conceptualized the project. GM and JRCB wrote the manuscript, with contributions from all authors. All authors contributed to the article and approved the submitted version.

\section{FUNDING}

This work was funded by a UBC Centre for Blood Research Post-doctoral transition award, and by a United Kingdom Biotechnology and Biological Sciences Research Council (BBSRC) grant (BB/R009759/2), both to JRCB. Cryo-EM data were collected at the UK national Electron Bio-Imaging center (eBIC), 
proposal EM19709-1, and at the University of Sheffield FoS Electron Microscopy Facility.

\section{ACKNOWLEDGMENTS}

We are grateful to Natalie Strynadka for use of her laboratory at the initial stage of the project, and to Susan Lea, Emily Furlong, and Steven Johnson for useful discussion on the FliF

\section{REFERENCES}

Berg, H. C. (2003). The rotary motor of bacterial flagella. Annu. Rev. Biochem. 72, 19-54. doi: 10.1146/annurev.biochem.72.121801.161737

Bergeron, J. R. C. (2016). Structural modeling of the flagellum MS ring protein FliF reveals similarities to the type III secretion system and sporulation complex. PeerJ 4:e1718. doi: 10.7717/peerj.1718

Bergeron, J. R. C., Brockerman, J. A., Vuckovic, M., Deng, W., Okon, M., Finlay, B. B., et al. (2018). Characterization of the two conformations adopted by the T3SS inner-membrane protein PrgK. Protein Sci. 27, 1680-1691. doi: 10.1002/pro.3447

Bergeron, J. R. C., Worrall, L. J., De, S., Sgourakis, N. G., Cheung, A. H., Lameignere, E., et al. (2015). The modular structure of the inner-membrane ring component PrgK facilitates assembly of the type III secretion system basal body. Structure 23, 161-172. doi: 10.1016/j.str.2014.10.021

Chaban, B., Hughes, H. V., and Beeby, M. (2015). The flagellum in bacterial pathogens: for motility and a whole lot more. Semin. Cell Dev. Biol. 46, 91-103. doi: 10.1016/j.semcdb.2015.10.032

Crepin, V. F., Prasannan, S., Shaw, R. K., Wilson, R. K., Creasey, E., Abe, C. M., et al. (2005). Structural and functional studies of the enteropathogenic Escherichia coli type III needle complex protein EscJ. Mol. Microbiol. 55, 1658-1670. doi: 10.1111/j.1365-2958.2005.04508.x

Dasgupta, N., Wolfgang, M. C., Goodman, A. L., Arora, S. K., Jyot, J., Lory, S., et al. (2003). A four-tiered transcriptional regulatory circuit controls flagellar biogenesis in Pseudomonas aeruginosa. Mol. Microbiol. 50, 809-824. doi: 10.1046/j.1365-2958.2003.03740.x

Duan, Q., Zhou, M., Zhu, L., and Zhu, G. (2013). Flagella and bacterial pathogenicity. J. Basic Microbiol. 53, 1-8. doi: 10.1002/jobm.201100335

Green, J. C. D., Kahramanoglou, C., Rahman, A., Pender, A. M. C., Charbonnel, N., and Fraser, G. M. (2009). Recruitment of the earliest component of the bacterial flagellum to the old cell division pole by a membrane-associated signal recognition particle family GTP-binding protein. J. Mol. Biol. 391, 679-690. doi: 10.1016/j.jmb.2009.05.075

Hendrixson, D. R., and DiRita, V. J. (2003). Transcription of sigma54-dependent but not sigma28-dependent flagellar genes in Campylobacter jejuni is associated with formation of the flagellar secretory apparatus. Mol. Microbiol. 50, 687-702. doi: 10.1046/j.1365-2958.2003.03731.x

Johnson, S., Fong, Y. H., Deme, J. C., Furlong, E. J., Kuhlen, L., and Lea, S. M. (2020). Symmetry mismatch in the MS-ring of the bacterial flagellar rotor explains the structural coordination of secretion and rotation. Nat. Microbiol. 5, 966-975. doi: 10.1038/s41564-020-0703-3

Johnson, S., Furlong, E. J., Deme, J. C., Nord, A. L., Caesar, J. J. E., Chevance, F. F. V., et al. (2021). Molecular structure of the intact bacterial flagellar basal body. Nat. Microbiol. 6, 712-721. doi: 10.1038/s41564-021-00895-y

Kawamoto, A., Miyata, T., Makino, F., Kinoshita, M., Minamino, T., Imada, K., et al. (2020). Native structure of flagellar MS ring is formed by 34 subunits with 23-fold and 11-fold subsymmetries. bioRxiv [Preprint]. doi: 10.1101/2020.10.11.334888

Kawamoto, A., and Namba, K. (2017). Structural study of the bacterial flagellar basal body by electron cryomicroscopy and image analysis. Methods Mol. Biol. 1593, 119-131. doi: 10.1007/978-1-4939-6927-2_9

Kazmierczak, B. I., and Hendrixson, D. R. (2013). Spatial and numerical regulation of flagellar biosynthesis in polarly flagellated bacteria. Mol. Microbiol. 88, 655-663. doi: 10.1111/mmi.12221

Kojima, S., Terashima, H., and Homma, M. (2020). Regulation of the single polar flagellar biogenesis. Biomol. Ther. 10:533. doi: 10.3390/biom10040533 symmetry. We thank John Hall (Wyatt) for assistance with the SEC-MALS data collection and analysis.

\section{SUPPLEMENTARY MATERIAL}

The Supplementary Material for this article can be found online at: https://www.frontiersin.org/articles/10.3389/fmicb.2021.781960/ full\#supplementary-material

Kubori, T., Shimamoto, N., Yamaguchi, S., Namba, K., and Aizawa, S. (1992) Morphological pathway of flagellar assembly in Salmonella typhimurium. J. Mol. Biol. 226, 433-446. doi: 10.1016/0022-2836(92)90958-m

Kusumoto, A., Shinohara, A., Terashima, H., Kojima, S., Yakushi, T., and Homma, M. (2008). Collaboration of FlhF and FlhG to regulate polar-flagella number and localization in Vibrio alginolyticus. Microbiology 154, 1390-1399. doi: 10.1099/mic.0.2007/012641-0

Kutsukake, K., Ohya, Y., and Iino, T. (1990). Transcriptional analysis of the flagellar regulon of Salmonella typhimurium. J. Bacteriol. 172, 741-747. doi: 10.1128/jb.172.2.741-747.1990

Levenson, R., Zhou, H., and Dahlquist, F. W. (2012). Structural insights into the interaction between the bacterial flagellar motor proteins FliF and FliG. Biochemistry 51, 5052-5060. doi: 10.1021/bi3004582

Li, N., Kojima, S., and Homma, M. (2011). Sodium-driven motor of the polar flagellum in marine bacteria vibrio. Genes Cells 16, 985-999. doi: 10.1111/j. 1365-2443.2011.01545.x

Li, H., and Sourjik, V. (2011). Assembly and stability of flagellar motor in Escherichia coli. Mol. Microbiol. 80, 886-899. doi: 10.1111/j.1365-2958.2011.07557.x

Macnab, R. M. (2003). How bacteria assemble flagella. Annu. Rev. Microbiol. 57, 77-100. doi: 10.1146/annurev.micro.57.030502.090832

Minamino, T., and Imada, K. (2015). The bacterial flagellar motor and its structural diversity. Trends Microbiol. 23, 267-274. doi: 10.1016/j. tim.2014.12.011

Minamino, T., Imada, K., and Namba, K. (2008). Molecular motors of the bacterial flagella. Curr. Opin. Struct. Biol. 18, 693-701. doi: 10.1016/j. sbi.2008.09.006

Morimoto, Y. V., Ito, M., Hiraoka, K. D., Che, Y.-S., Bai, F., Kami-Ike, N., et al. (2014). Assembly and stoichiometry of FliF and FlhA in Salmonella flagellar basal body. Mol. Microbiol. 91, 1214-1226. doi: 10.1111/mmi.12529

Morimoto, Y. V., and Minamino, T. (2014). Structure and function of the bidirectional bacterial flagellar motor. Biomol. Ther. 4, 217-234. doi: 10.3390/ biom4010217

Nakamura, S., and Minamino, T. (2019). Flagella-driven motility of bacteria. Biomol. Ther. 9:279. doi: 10.3390/biom9070279

Niehus, E., Gressmann, H., Ye, F., Schlapbach, R., Dehio, M., Dehio, C., et al. (2004). Genome-wide analysis of transcriptional hierarchy and feedback regulation in the flagellar system of Helicobacter pylori. Mol. Microbiol. 52, 947-961. doi: 10.1111/j.1365-2958.2004.04006.x

Pandza, S., Baetens, M., Park, C. H., Au, T., Keyhan, M., and Matin, A. (2000). The G-protein FlhF has a role in polar flagellar placement and genera stress response induction in Pseudomonas putida. Mol. Microbiol. 36, 414-423. doi: 10.1046/j.1365-2958.2000.01859.x

Rohou, A., and Grigorieff, N. (2015). CTFFIND4: fast and accurate defocus estimation from electron micrographs. J. Struct. Biol. 192, 216-221. doi: 10.1016/j.jsb.2015.08.008

Scheres, S. H. W. (2012). RELION: implementation of a Bayesian approach to cryo-EM structure determination. J. Struct. Biol. 180, 519-530. doi: 10.1016/j. jsb.2012.09.006

Scheres, S. H. W. (2020). Amyloid structure determination in RELION-3.1. Acta Crystallogr. Sect. F Struct. Biol. Cryst. Commun. 76, 94-101. doi: 10.1107/ S2059798319016577

Schuhmacher, J. S., Rossmann, F., Dempwolff, F., Knauer, C., Altegoer, F., Steinchen, W., et al. (2015a). MinD-like ATPase FlhG effects location and number of bacterial flagella during C-ring assembly. Proc. Natl. Acad. Sci. U. S. A. 112, 3092-3097. doi: 10.1073/pnas.1419388112 
Schuhmacher, J. S., Thormann, K. M., and Bange, G. (2015b). How bacteria maintain location and number of flagella? FEMS Microbiol. Rev. 39, 812-822. doi: 10.1093/femsre/fuv034

Spreter, T., Yip, C. K., Sanowar, S., André, I., Kimbrough, T. G., Vuckovic, M., et al. (2009). A conserved structural motif mediates formation of the periplasmic rings in the type III secretion system. Nat. Struct. Mol. Biol. 16, 468-476. doi: 10.1038/nsmb.1603

Suzuki, H., Yonekura, K., and Namba, K. (2004). Structure of the rotor of the bacterial flagellar motor revealed by electron cryomicroscopy and singleparticle image analysis. J. Mol. Biol. 337, 105-113. doi: 10.1016/j.jmb.2004.01.034

Takekawa, N., Kawamoto, A., Sakuma, M., Kato, T., Kojima, S., Kinoshita, M., et al. (2021). Two distinct conformations in $34 \mathrm{FliF}$ subunits generate three different symmetries within the flagellar MS-ring. MBio 12:e03199-20. doi: 10.1128/mBio.03199-20

Terashima, H., Hirano, K., Inoue, Y., Tokano, T., Kawamoto, A., Kato, T., et al. (2020). Assembly mechanism of a supramolecular MS-ring complex to initiate bacterial flagellar biogenesis in vibrio species. J. Bacteriol. 202:e00236-20. doi: $10.1128 /$ JB.00236-20

Wagner, T., Merino, F., Stabrin, M., Moriya, T., Antoni, C., Apelbaum, A., et al. (2019). SPHIRE-crYOLO is a fast and accurate fully automated particle picker for cryo-EM. Commun. Biol. 2, 218-213. doi: 10.1038/s42003019-0437-z

Yip, C. K., Kimbrough, T. G., Felise, H. B., Vuckovic, M., Thomas, N. A., Pfuetzner, R. A., et al. (2005). Structural characterization of the molecular platform for type III secretion system assembly. Nature 435, 702-707. doi: 10.1038/nature03554

Yonekura, K., Maki-Yonekura, S., and Namba, K. (2002). Growth mechanism of the bacterial flagellar filament. Res. Microbiol. 153, 191-197. doi: 10.1016/ s0923-2508(02)01308-6
Zeng, H., Wang, S., Zhou, T., Zhao, F., Li, X., Wu, Q., et al. (2018). ComplexContact: a web server for inter-protein contact prediction using deep learning. Nucleic Acids Res. 46, W432-W437. doi: 10.1093/nar/gky420

Zeytuni, N., Hong, C., Flanagan, K. A., Worrall, L. J., Theiltges, K. A., Vuckovic, M., et al. (2017). Near-atomic resolution cryoelectron microscopy structure of the 30-fold homooligomeric SpoIIIAG channel essential to spore formation in Bacillus subtilis. Proc. Natl. Acad. Sci. U. S. A. 114, E7073-E7081. doi: 10.1073/pnas.1704310114

Zheng, S. Q., Palovcak, E., Armache, J.-P., Verba, K. A., Cheng, Y., and Agard, D. A. (2017). MotionCor2: anisotropic correction of beam-induced motion for improved cryo-electron microscopy. Nat. Methods 14, 331-332. doi: $10.1038 /$ nmeth.4193

Conflict of Interest: The authors declare that the research was conducted in the absence of any commercial or financial relationships that could be construed as a potential conflict of interest.

Publisher's Note: All claims expressed in this article are solely those of the authors and do not necessarily represent those of their affiliated organizations, or those of the publisher, the editors and the reviewers. Any product that may be evaluated in this article, or claim that may be made by its manufacturer, is not guaranteed or endorsed by the publisher.

Copyright (C) 2022 Mariano, Faba-Rodriguez, Bui, Zhao, Ross, Tzokov and Bergeron. This is an open-access article distributed under the terms of the Creative Commons Attribution License (CC BY). The use, distribution or reproduction in other forums is permitted, provided the original author(s) and the copyright owner(s) are credited and that the original publication in this journal is cited, in accordance with accepted academic practice. No use, distribution or reproduction is permitted which does not comply with these terms. 